\title{
Accumulation of advanced glycation end products potentiate human retinal capillary endothelial cells mediated diabetic retinopathy
}

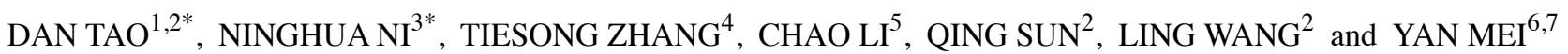 \\ ${ }^{1}$ College of Environmental Science and Engineering, Kunming University of Science and Technology, Kunming, Yunnan 650500; \\ ${ }^{2}$ Department of Ophthalmology, Kunming Children's Hospital; ${ }^{3}$ Department of Ophthalmology, \\ The First People's Hospital of Yunnan Province, Kunming, Yunnan 650031; \\ Departments of ${ }^{4}$ Otolaryngology and ${ }^{5}$ Anesthesiology, Kunming Children's Hospital, Kunming, Yunnan 650228; \\ ${ }^{6}$ School of Medicine, Kunming University of Science and Technology, Kunming, Yunnan 650500; \\ ${ }^{7}$ The First People's Hospital of Yunnan Province, Kunming, Yunnan 650031, P.R. China
}

Received December 1, 2017; Accepted September 7, 2018

DOI: $10.3892 / \mathrm{mmr} .2019 .10590$

\begin{abstract}
The major ophthalmic complication in patients with diabetes is diabetic retinopathy (DR), which is one of the major eye diseases that causes blindness. It is well established that the occurrence and duration of DR is positively correlated with duration of diabetes. Advanced glycation end product (AGE) accumulation in patients with diabetes is one factor that leads to the development of DR. However, the underlying mechanisms remain unclear. In the present study, the role of phosphoinositide 3-kinase/protein kinase B (Akt) signaling in AGE-induced DR development was investigated. An in vitro experimental system was used to study the effects of AGEs on human retinal capillary endothelial cells (HRCECs) and Müller cells. Flow cytometry, MTT, western blotting and BrdU incorporation assays were performed. Reverse transcription-quantitative polymerase chain reaction was used to measure the expression of angiogenesis-associated genes. Functional assays of angiogenesis, including HRCEC invasion and tube formation assays. It was demonstrated that the expression of receptor for AGEs was upregulated in HRCECs and Müller cells following treatment with AGEs. AGE treatment did not affect Müller cell viability, but enhanced HRCEC viability. Akt inhibition increased cell apoptosis and death in HRCECs. AGE treatment upregulated the expression of pro-angiogenic genes, which was suppressed by Akt inhibitor
\end{abstract}

Correspondence to: Dr Yan Mei, School of Medicine, Kunming University of Science and Technology, 68 Wenchang Road, Yieryi Avenue, Kunming, Yunnan 650500, P.R. China

E-mail: yan_ym665@163.com

*Contributed equally

Key words: advanced glycation end products, diabetic retinopathy, human retinal capillary endothelial cells, protein kinase $\mathrm{B}$, angiogenesis treatment. In addition, Akt inhibitor treatment suppressed HRCEC invasion and tube formation ability. The present study suggested that Akt-mediated signaling may serve critical roles in the development of DR due to the accumulation of AGEs. Akt may be a potential therapeutic target in DR.

\section{Introduction}

Patients with diabetes mellitus and chronic hyperglycemia are at risk of developing complications, including diabetic retinopathy (DR), nephropathy, neuropathy, cardiomyopathy, rheumatoid arthritis and osteoporosis $(1,2)$. The pathogenesis of these complications is strongly associated with the glycation of plasma proteins, which produces a large number of advanced glycation end products (AGEs) $(3,4)$. Protein glycation interferes with their physiological function by altering molecular conformation, enzymatic activity and receptor functioning. AGEs interact with their membrane-localized receptors [receptor for advanced glycation end products (RAGE)] and alter intracellular signaling to influence various biological processes within cells $(5,6)$. The mechanisms by which accumulation of AGEs cause DR has not yet been extensively studied.

Since its initial discovery as a proto-oncogene, protein kinase $\mathrm{B}$ (Akt) has become a major focus of attention, due to its critical involvement in cell apoptosis regulation, angiogenesis, autophagy, transcription, protein synthesis and glucose metabolism (7). The Akt signaling cascade is activated by multiple cell receptors, including receptor tyrosine kinases, B and $\mathrm{T}$ cell receptors, cytokine receptors and G-protein coupled receptors (7). Other stimuli that induce the production of phosphatidylinositol $(3,4,5)$-trisphosphates via phosphoinositide 3-kinase (PI3K) also induce activation of the Akt signaling cascade (7). Inhibition of the PI3K/Akt/mammalian target of rapamycin pathway has potential therapeutic function in DR pathophysiology (8). However, whether the PI3K/Akt pathway influences the function of AGEs during the development of DR remains unclear. In the present study, the mechanisms of 
AGEs in DR were investigated. Specifically, the role of Akt signaling in DR pathophysiology was examined.

\section{Materials and methods}

Cell culture. Primary human retinal capillary endothelial cells (HRCECs) and primary human Müller cells were purchased from the Type Culture Collection of the Chinese Academy of Sciences (Shanghai, China). Müller cells were cultured in high-glucose Dulbecco's modified Eagle's medium (Thermo Fisher Scientific, Inc., Waltham, MA, USA) supplemented with $100 \mathrm{U} / \mathrm{ml}$ penicillin, $100 \mu \mathrm{g} / \mathrm{ml}$ streptomycin and $10 \%$ fetal bovine serum (FBS; Thermo Fisher Scientific, Inc.). HRCECs were cultured in RMPI-1640 medium (Thermo Fisher Scientific, Inc.) with the same supplements. Cells were sub-cultured until $80 \%$ confluence was reached. A gradient of AGEs conjugated to bovine serum albumin $(0,25,50$ and $100 \mu \mathrm{g} / \mathrm{ml}$; cat. no. 121800; Sigma-Aldrich; Merck KGaA, Darmstadt, Germany) was used to treat the cells for $24 \mathrm{~h}$ at $37^{\circ} \mathrm{C}$. LY294002 (1 $\mu \mathrm{M}$; Sigma-Aldrich; Merck KGaA), a PI3K inhibitor (PI3Ki), was also used to treat cells for $6 \mathrm{~h}$ at $37^{\circ} \mathrm{C}$. Akt inhibitor (Akti; $5 \mu \mathrm{M}$; Akt $1 / 2$ kinase inhibitor; cat. no. sc-300173; Santa Cruz Biotechnology, Inc., Dallas, TX, USA) was used to block Akt function in cells (cells were treated for $6 \mathrm{~h}$ at $\left.37^{\circ} \mathrm{C}\right)$.

MTT assay. Following treatment, cell media was removed by careful aspiration. Subsequently, $50 \mu 1$ serum-free media and $50 \mu 1$ MTT solution was added into each well $\left(10^{4}\right.$ per $\left.\mathrm{ml}\right)$. The plate was incubated at $37^{\circ} \mathrm{C}$ for $3 \mathrm{~h}$. Following incubation, $150 \mu 1$ MTT solvent (DMSO) was added into each well. The plate was then warmed in foil and shaken on an orbital shaker for $15 \mathrm{~min}$. The plate was read at an absorbance of $590 \mathrm{~nm}$ after being maintained in the incubator for $1 \mathrm{~h}$.

Western blotting. In brief, cells were collected and lysed in radioimmunoprecipitation assay lysis buffer with proteinase inhibitor $(20 \mathrm{nM})$ and phosphatase inhibitor $(20 \mathrm{nM}$; Thermo Fisher Scientific, Inc.). The extracted protein was measured and normalized with a bicinchoninic acid protein assay. Equivalent amounts of total proteins (30 $\mu \mathrm{g}$ per lane) were subsequently separated by electrophoresis on 4-20\% gradient SDS-PAGE gels and were transferred to a polyvinylidene fluoride membrane by a half-dry transferring system. Blocking was performed by incubating the membrane with 5\% BSA in TBST for $15 \mathrm{~min}$ at room temperature. The following primary antibodies were used for target proteins: RAGE (1:1,000; cat. no. ab37647; Abcam, Cambridge, MA, USA), Akt (1:1,000; cat. no. ab126811; Abcam), phosphorylated (p)-Akt (1:1,000; cat. no. ab81283; Abcam), PI3K (1:1,000; cat. no. ab86714; Abcam), and p-PI3K (1:1,000; cat. no. ab182651; Abcam) and GAPDH (1:1,000; cat. no. ab181602; Abcam). The primary antibodies were incubated with the polyvinylidene fluoride membrane at $4^{\circ} \mathrm{C}$ overnight. Horseradish peroxidase-conjugated secondary antibody (1:5,000; cat. nos. ab6721 and ab6728; Abcam) were added to the membrane to incubated for $1 \mathrm{~h}$ at room temperature. Enhanced chemiluminescence western blotting detection reagents (Thermo Fisher Scientific, Inc.) were used for imaging. ImageJ software 1.52a (National Institutes of Health, Bethesda, MD, USA) was used for densitometry.
Invasion assay. Transwells coated with Matrigel (Corning Incorporated, Corning, NY, USA) with an $8 \mu \mathrm{m}$ polycarbonate filter membrane were used for the invasion assay. HRCECs $\left(3 \times 10^{4}\right)$ were added into the upper chamber with serum-free medium (cat. no. 211; Sigma-Aldrich; Merck KGaA). The lower chamber was filled with 5\% serum medium (cat. no. 211; Sigma-Aldrich; Merck KGaA). HRCECs were subsequently treated with AGEs $(100 \mu \mathrm{g} / \mathrm{ml})$ and Akti $(5 \mu \mathrm{M})$ for a $24 \mathrm{~h}$ incubation at $37^{\circ} \mathrm{C}$. Cells on the top surface of the filter were removed, and the remaining cells on the underside of the filter were subsequently fixed with the fixation buffer $(0.1 \mathrm{M}$ sodium cacodylate buffer supplemented with $4 \%$ paraformaldehyde, $2.5 \%$ glutaraldehyde and $0.02 \%$ picric acid) at room temperature for $1 \mathrm{~h}$, and stained with $5 \%$ crystal violet for $10 \mathrm{~min}$ at room temperature. The stained membrane was washed with PBS three times and the invaded cell number on the membrane was counted using a light microscope.

BrdU incorporation assay. BrdU incorporation assay was performed to assess the cell proliferation. A commercial BrdU cell proliferation assay kit (cat. no. 6813; Cell Signaling Technology, Inc., Danvers, MA, USA) was used and the manufacture's protocol was carefully followed. Briefly, $5 \times 10^{4}$ cells were seeded in 96 -well plate $24 \mathrm{~h}$ before the $\mathrm{BrdU}$ incorporation assay. Then BrdU solution was added to the plate well and incubated for $12 \mathrm{~h}$ at $37^{\circ} \mathrm{C}$. Then, the cells were collected by centrifuging the plate at $300 \mathrm{x}$ g for $10 \mathrm{~min}$ at $4^{\circ} \mathrm{C}$. Fixing/denaturing solution was added to each well $(100 \mu \mathrm{l} /$ well $)$ and the plate was kept at room temperature for $30 \mathrm{~min}$. Following the removal of the fixing/denaturing solution, detection antibody solution from the kit was added for incubation at room temperature for $1 \mathrm{~h}$. HRP-conjugated secondary antibody solution was added to incubate at room temperature for $30 \mathrm{~min}$. Absorbance at $450 \mathrm{~nm}$ was read after the stop solution was added.

Flow cytometry. To detect cell apoptosis and necrosis, an Annexin $\mathrm{V}$ and propidium iodide (PI) staining method was utilized. A commercial kit (cat. no. V1324; Thermo Fisher Scientific, Inc.) was used in accordance with the manufacturer's protocols. Cells were washed twice with cold PBS and subsequently resuspended in $1 \mathrm{X}$ binding buffer. Then, $10^{5}$ cells in $100 \mu 1$ annexin-binding buffer were transferred to a $5 \mathrm{ml}$ culture tube and $1 \mu \mathrm{l}$ annexin $\mathrm{V}$-fluorescein isothiocyanate (FITC) and $2 \mu \mathrm{l}$ PI was added to the tube. Samples were gently mixed and incubated for $15 \mathrm{~min}$ at room temperature in the dark. Subsequently, $400 \mu 11 \mathrm{x}$ binding buffer was added to each tube. Samples were analyzed by flow cytometry within $30 \mathrm{~min}$ following staining. A FACSCanto II machine (BD Biosciences, Franklin Lakes, NJ, USA) was used.

In additon, the expression of glial fibrillary acidic protein (GFAP) on Müller cells and cluster of differentiation (CD)34 protein on HRCECs was measured by flow cytometry. Cell pellets of Müller cells and HRCECs were harvested following centrifugation at $500 \mathrm{x} \mathrm{g}$ and $5 \mathrm{~min}$ at $4^{\circ} \mathrm{C}$. Then an equal number $\left(10^{5}\right)$ of these cells were collected for subsequent steps. They were blocked with PBS with 5\% bovine serum albumin (BSA; cat. no. A2058; Sigma-Aldrich; Merck KGaA) for $10 \mathrm{~min}$ at room temperature. Then mouse antibodies of 
Table I. Primers used for reverse transcription-quantitative polymerase chain reaction.

\begin{tabular}{lll}
\hline Gene & \multicolumn{1}{c}{ Forward primer $\left(5^{\prime}-3^{\prime}\right)$} & Reverse primer $\left(5^{\prime}-3^{\prime}\right)$ \\
\hline $18 S$ rRNA & CTACCACATCCAAGGAAGCA & TTTTTCGTCACTACCTCCCCG \\
VEGF & GTCCGATTGAGACCCTGGTG & ACCGGGATTTCTTGCGCTTT \\
VEGFR & GTGTCTATAGGTGCCGAGCC & CGGAAGAAGACCGCTTCAGT \\
Ang1 & TCAGCCTTTGCACTAAAGAAGTTT & GGCCCTTTGAAGTAGTGCCA \\
Ang 2 & AAGGAAGCCCTTATGGACGA & CCAGCCATTCTCACAGCCAA \\
FGF & CACTTTCCCAGGAGGATGGAG & TCCCCAGCTGAGAAGACACT \\
PDGF & TACTGAATTTCGCCGCCACA & GGAGGAGAACAAAGACCGCA \\
PEDF & TACTCCTCTGGACTGGAGCC & TGGATCTCAGGCGGTACAGA
\end{tabular}

rRNA, ribosomal RNA; VEGF, vascular endothelial growth factor; VEGFR, vascular endothelial growth factor receptor; PEDF, pigment epithelium-derived factor; Ang1, angiopoietin 1; Ang2, angiopoietin 2; FGF, fibroblast growth factor; PDGF, platelet-derived growth factor.

GFAP (1:200; cat. no. G3893, Sigma-Aldrich; Merck KGaA) and CD34 (1:200; cat. no. SAB4700160, Sigma-Aldrich; Merck KGaA) were incubated with Müller cells and HRCECs for $10 \mathrm{~min}$ at room temperature, respectively. To measure the expression of cleaved caspase- 3 , the $10^{5}$ harvested HRCECs and Müller cells were washed in cold PBS once, and $2 \mathrm{ml}$ cold 4\% paraformaldehyde (Sigma-Aldrich; Merck KGaA). Following washing with cold PBS for 5 min, permeabilization buffer (cat. no. 22016, Biotium, Inc.) was added to incubate at $4^{\circ} \mathrm{C}$ for $30 \mathrm{~min}$. Cells were washed in PBS prior to the addition of cleaved caspase-3 (1:100; cat. no. ab2302, Abcam) antibodies to the cells. Following incubation with GFAP, CD34 or cleaved caspase- 3 antibodies for $30 \mathrm{~min}$ at $4^{\circ} \mathrm{C}$, the cells were washed by PBS. Subsequently, FITC-labeled anti-mouse secondary antibodies (1:5,000, cat. no. SAB3701081; Sigma-Aldrich; Merck KGaA) and APC-preadsorbed anti-rabbit secondary antibodies (1:5,000, cat. no. ab130805, Abcam) were added to the cells to incubate for $20 \mathrm{~min}$ at room temperature. Following three washes in PBS, cells were analyzed using the FACSCanto II machine (BD Biosciences) and FlowJo software (version 10.0.7, FlowJo LLC, Ashland, OR, USA) was used to analyze the data.

Tube formation assay. Prior to the tube formation assay, HRCECs $\left(1 \times 10^{5}\right.$ cells/well) were starved in medium containing $1 \% \mathrm{FBS}$ for $12 \mathrm{~h}$ at $37^{\circ} \mathrm{C}$. Cells were incubated with different concentrations of AGEs for another $12 \mathrm{~h}$ at $37^{\circ} \mathrm{C}$. Cells were subsequently seeded into 24 well plates pre-coated with Matrigel (BD Biosciences) and treated with AGEs $(50 \mu \mathrm{g} / \mathrm{ml})$ or Akti $(5 \mu \mathrm{M})$ for $8 \mathrm{~h}$ at $37^{\circ} \mathrm{C}$. Images were captured under an inverted microscope at 200x magnification and 5 fields were randomly selected for assessment.

Reverse transcription-quantitative polymerase chain reaction $(R T-q P C R)$. PCR was performed to detect expression of vascular endothelial growth factor (VEGF), VEGF receptor (VEGFR), pigment epithelium-derived factor (PEDF), angiopoietin (Ang) 1, Ang 2, fibroblast growth factor (FGF), platelet-derived growth factor (PDGF) and 18S rRNA. The primers used for these genes are listed in Table I. RNA was isolated from the cell lines according to the manufacturer's protocol using TRIzol (Invitrogen; Thermo Fisher Scientific,
Inc.). Total RNA ( $1 \mu \mathrm{g})$ was reverse transcribed using High Capacity cDNA Reverse Transcription kit (Thermo Fisher Scientific, Inc.). PCR was performed on a LightCycler II 480 (Roche Diagnostics, Basel, Switzerland) with SYBR Green I dye (Roche Diagnostics). The results were normalized to the housekeeping 18S rRNA gene.

Statistical analysis. Statistical analyses were performed using GraphPad Prism 5.0 (GraphPad Software, Inc., CA, USA). All experiments were repeated 3 times. Data were expressed as the mean \pm standard error of the mean. Two-tailed Student's t-test was used to evaluate the significance of differences between two groups. One-way analysis of variance was used to compare results with more than three groups. Tukey's post-hoc test was performed for multiple comparisons. $\mathrm{P}<0.05$ was considered to indicate a statistically significant difference.

\section{Results}

RAGE expression is upregulated in HRCECs and Müller cells in response to AGE treatment. To investigate the effects of AGEs on DR, primary Müller cells (Fig. 1A) and HRCECs (Fig. 1B) were cultured in vitro, and the expression of RAGE was measured. Biomarkers of human retinal capillary endothelial cells (CD34) and Muller cells (GFAP) were assessed by flow cytometry. These two biomarkers were highly expressed in the two kinds of cells, respectively (Fig. 1C and D). The results revealed that there was a basal level of RAGE expression in Müller cells (Fig. 1E) and HRCECs (Fig. 1F). Following AGE treatment at $100 \mu \mathrm{g} / \mathrm{ml}$, RAGE expression was significantly upregulated in the two cell cultures by more than five-fold, compared with the untreated cells $(\mathrm{P}<0.0001$; Fig. $1 \mathrm{G}$ and $\mathrm{H})$. The increase in RAGE expression was proportional to the concentration of AGE treatment. These data provided initial verification that Müller cells and HRCECs were responsive to AGE treatment.

Akt inhibition reduces HRCEC viability. As the expression of RAGE was upregulated by the AGE treatment in Müller cells and HRCECs, the biological effects of AGE treatment $(100 \mu \mathrm{g} / \mathrm{ml})$ were examined. The cell viability assay results demonstrated that the AGE treatment did not exert 

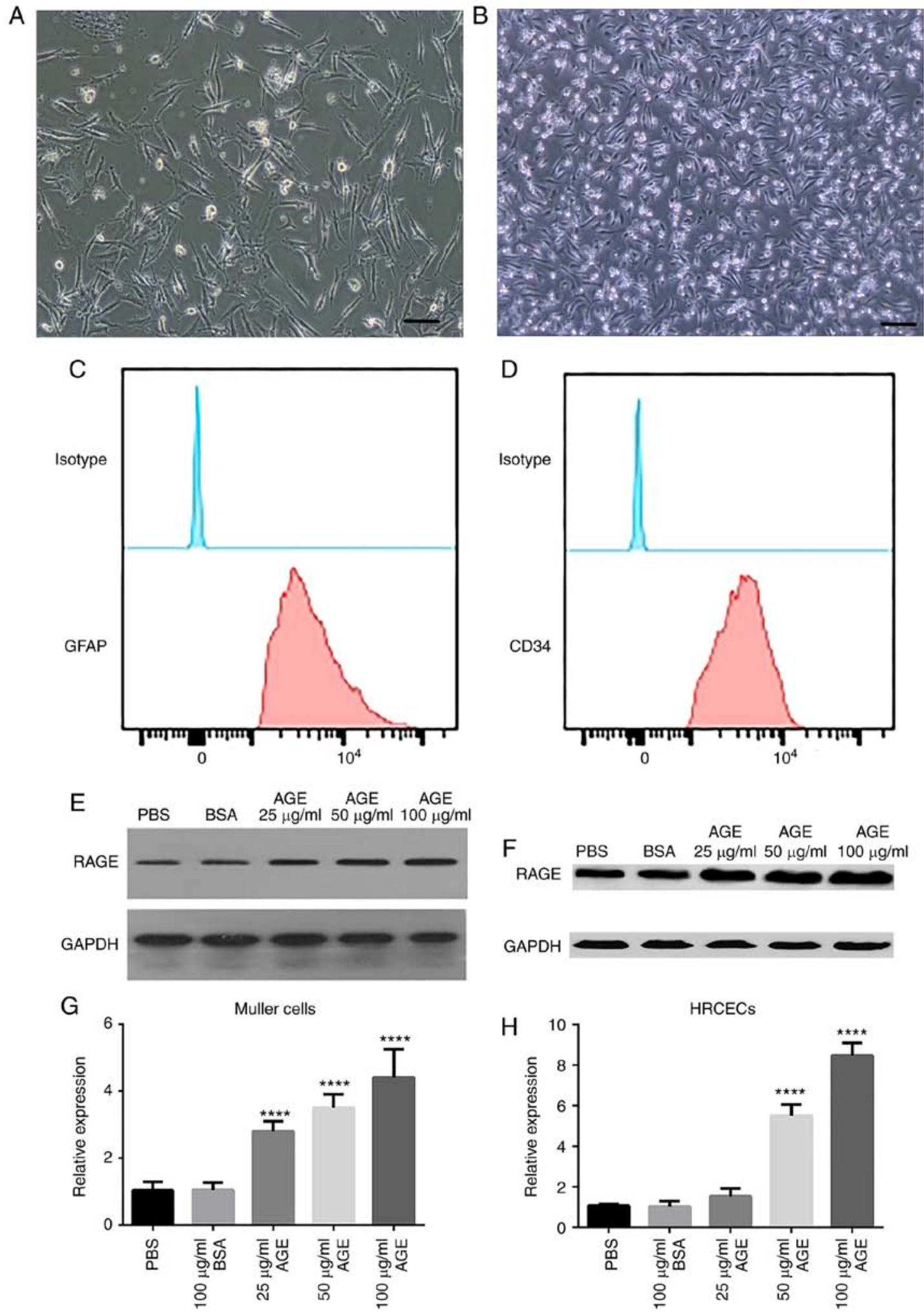

Figure 1. RAGE expression in Müller cells and HRCECs. (A) Morphological characteristics of Müller cells and (B) HRCECs observed under a light microscope (the black scale bar, $100 \mu \mathrm{m}$ ). (C) Representative histograms of flow cytometry data for the detection of GFAP protein in Müller cells and (D) CD 34 protein in HRCECs. (E) AGE treatment upregulated the expression of RAGE in Müller cells and (F) HRCECs. (G) The difference between the treated groups and the untreated groups was statistically significant in Müller cells and (H) HRCECs. ${ }^{* * *} \mathrm{P}<0.001,{ }^{* * * *} \mathrm{P}<0.0001$ vs. the control group (AGE treatment replaced by PBS). RAGE, receptor for advanced glycation end products; HRCECs, human retinal capillary endothelial cells; GFAP, glial fibrillary acidic protein; AGE, advanced glycation end products; BSA, bovine serum albumin; $\mathrm{CD}$, cluster of differentiation.

a significant effect on Müller cell viability (Fig. 2A), but enhanced HRCEC viability (Fig. 2B). The PI3K/Akt pathway has a profound impact on cell proliferation and survival (8).
Müller cells and HRCECs were treated with PI3Ki and Akti, and it was noticed that Akti significantly reduced the effects induced by AGE treatment $(\mathrm{P}<0.0001$; Fig. $2 \mathrm{~B})$, although 
A

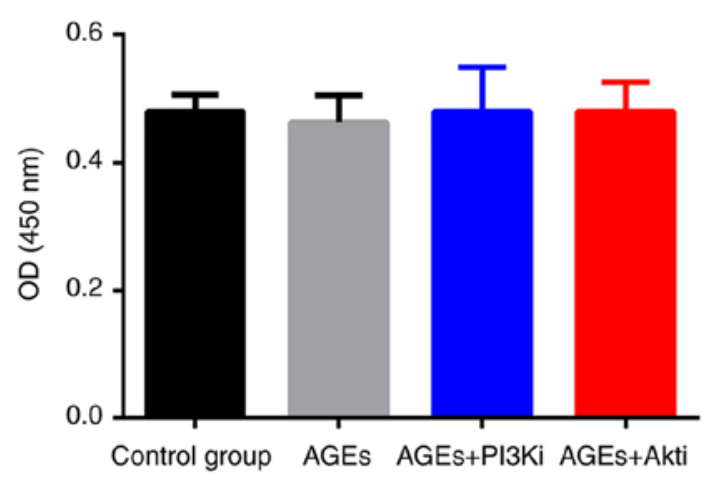

C

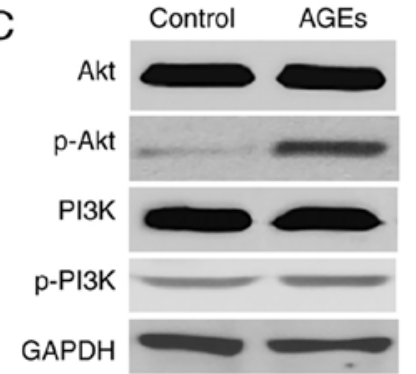

B

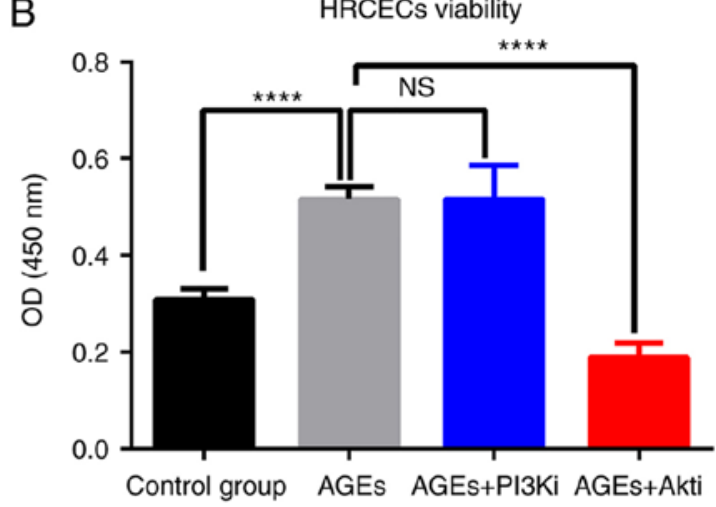

D

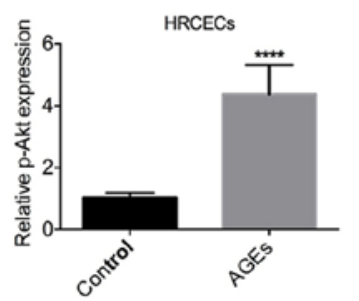

Figure 2. AGE treatment increases HRCEC viability. (A) Müller cell viability was not altered by treatment. (B) AGE treatment enhanced HRCEC viability, whereas Akti treatment suppressed it. (C) AGE treatment stimulated Akt phosphorylation, but not PI3K phosphorylation. (D) The differences in Akt phosphorylation were statistically significant, but that of the PI3K phosphorylation was not. ${ }^{* * * *} \mathrm{P}<0.0001$ vs. control group or the AGEs treated group. AGE, advanced glycation end products; HRCEC, human retinal capillary endothelial cell; PI3K, phosphoinositide 3-kinase; Akt, protein kinase B; p, phosphorylated; i, inhibitor; NS, not significant.

PI2Ki did not notably influence the effect of AGE treatment. Western blotting analysis confirmed that AGE treatment upregulated Akt phosphorylation, but not PI3K phosphorylation (Fig. 2C and D).

Akt inhibition suppresses HRCEC proliferation and induces apoptosis. To understand the mechanisms underlying the alterations in cell viability as a result of AGE and Akti treatment in HRCECs, cell proliferation and apoptosis was measured via a BrdU incorporation assay and flow cytometry, respectively. Flow cytometry demonstrated that Akti treatment markedly increased the cell death rate in HRCECs (Fig. 3A). The expression of full length and cleaved caspase-3 was also quantified in Müller cells and HRCECs following treatment. As presented in Fig. 3B, neither the AGE treatment nor the Akti treatment stimulated cell apoptosis in Müller cells. However, in HRCECs, the Akti treatment induced cleaved caspase-3 expression (Fig. 3B). The BrdU incorporation assay detected cell proliferation. In HRCECs, AGE treatment significantly increased cell proliferation, and Akti treatment suppressed cell proliferation (Fig. 3C). These results indicated that AGE treatment enhanced HRCEC viability by increasing cell proliferation, and that Akti treatment reduced HRCEC viability via suppressing cell proliferation, as well as via induction of apoptosis.

Akt inhibition reduces angiogenesis-associated gene expression. The angiogenic function of HRCECs was further investigated by quantifying the relative expression of angiogenesis-associated genes using RT-qPCR, including the pro-angiogenic genes Ang1, Ang2, VEGF, VEGFR, PDGF and FGF, as well as the anti-angiogenic gene PEDF.
The expression level was normalized by calculating the $\mathrm{z}$ scores. Expression levels of these pro-angiogenic genes were upregulated by AGEs treatment, whereas PEDF expression was downregulated (Fig. 4). Furthermore, Akti treatment suppressed pro-angiogenic gene expression induced AGE treatment.

Akt inhibition suppresses the tube formation ability of $H R C E C s$. In addition to measuring the expression of angiogenesis-associated genes, functional assays were performed to further assess the HRCEC angiogenesis. In the in vitro invasion assay, HRCECs treated with AGE $(100 \mu \mathrm{g} / \mathrm{ml})$ had the highest invasive ability (Fig. 5A), whereas the AGEs and Akti-treated HRCECs exhibited a comparable invasive ability to control cells (Fig. 5A). A similar trend was observed in the tube formation assay (Fig. 5B). Quantitative analysis revealed that these differences were significant $(\mathrm{P}<0.01$; Fig. 5C and D). These data, together with the angiogenic gene expression data, suggested that AGEs had a pro-angiogenic effect in HRCECs, and Akt inhibition had an anti-angiogenic effect.

\section{Discussion}

Patients with a long history of diabetes mellitus frequently have comorbidities, including DR and cardiomyopathy, which are highly associated with a chronic hyperglycemic state $(1,2,9)$. Abnormal, newly formed blood vessels grow from the retina and result in subsequent tractional retinal detachment and hemorrhage, which is the major concern of DR (9-11). Although the underlying mechanisms of aberrant angiogenesis in DR remains unclear, evidence has demonstrated AGE 


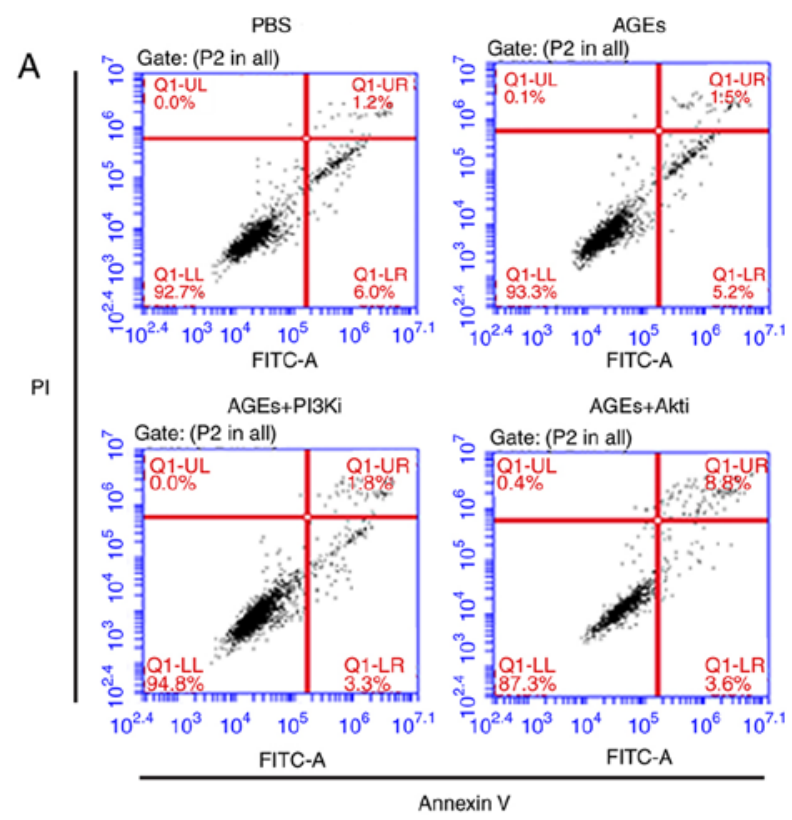

B
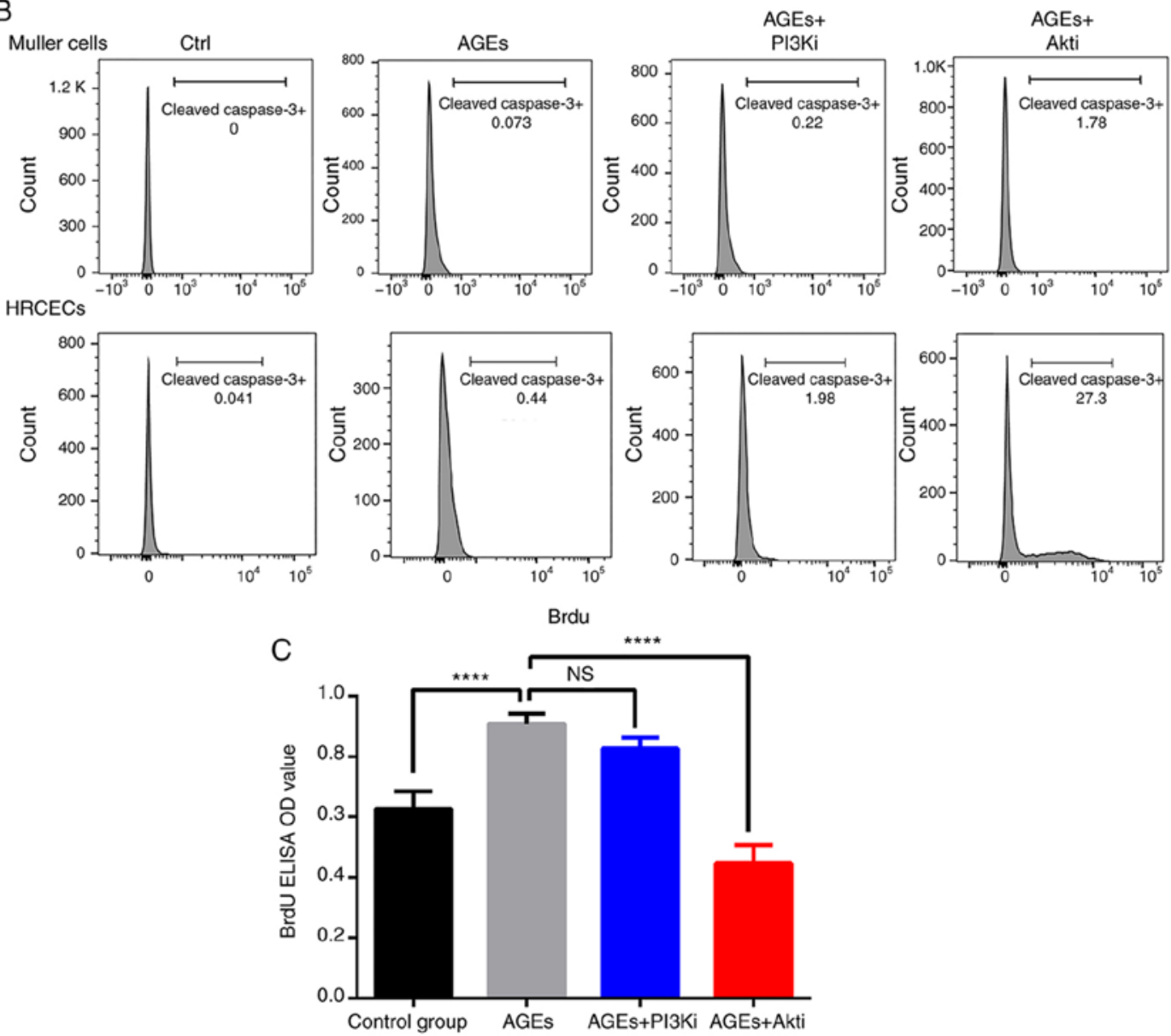

Figure 3. Akti induces HRCEC apoptosis and suppresses proliferation. (A) Flow cytometry analysis of HRCEC apoptosis when treated with AGE, PI3Ki and Akti. Akti treatment increased the number of apoptotic cells (the upper right quadrant was designated as apototic cells). (B) Flow cytometry analysis of cleaved caspase-3 expression in treated HRCECs and Müller cells. (C) BrdU ELISA indicated that AGE treatment increased HRCEC proliferation, and Akt inhibitor treatment decreased HRCEC proliferation. ${ }^{* * * *} \mathrm{P}<0.0001$ vs. AGEs treated group. Ctrl, control; BSA, bovine serum albumin; ELISA, enzyme-linked immunosorbent assay; Q, quadrant; FITC, fluorescein isothiocyanate; PI, propidium iodide; AGE, advanced glycation end products; HRCECs, human retinal capillary endothelial cells; NS, not significant; OD, optical density; PI3K, phosphoinositide 3-kinase; Akt, protein kinase B; i, inhibitor.

accumulation is a major factor that contributes to angiogenesis (12). AGEs interact with RAGEs, therefore altering intracellular signaling and influencing several essential biological processes within cells (3-6). The exact pathological mechanisms of AGE-induced angiogenesis in DR remain to be elucidated. 


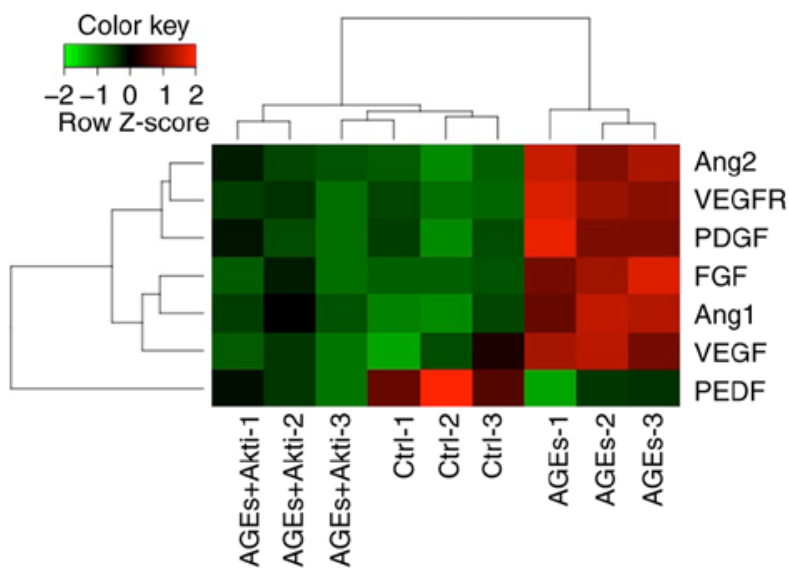

Figure 4. Expression of angiogenesis-associated genes in human retinal capillary endothelial cells. mRNA expression of was plotted as a heatmap. Red indicates relatively high expression level, and green indicates a relatively low expression level. AGE, advanced glycation end products; Ctrl, control; VEGF, vascular endothelial growth factor; VEGFR, vascular endothelial growth factor receptor; PEDF, pigment epithelium-derived factor; Ang1, angiopoietin 1; Ang2, angiopoietin 2; FGF, fibroblast growth factor; PDGF, platelet-derived growth factor; PI3K, phosphoinositide 3-kinase; Akt, protein kinase B; i, inhibitor; Ctrl, control.

A

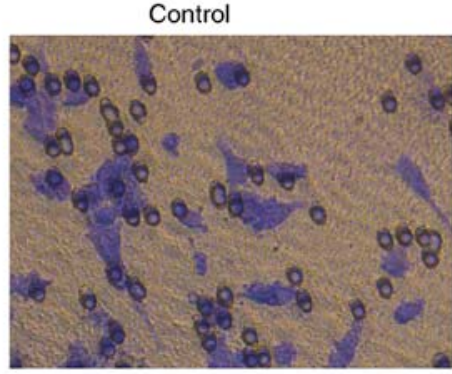

B

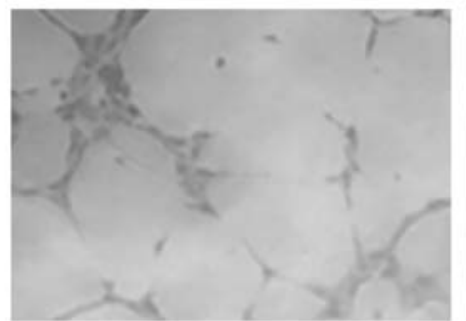

C

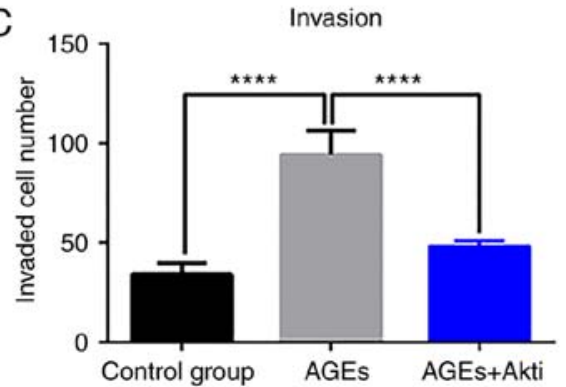

AGEs
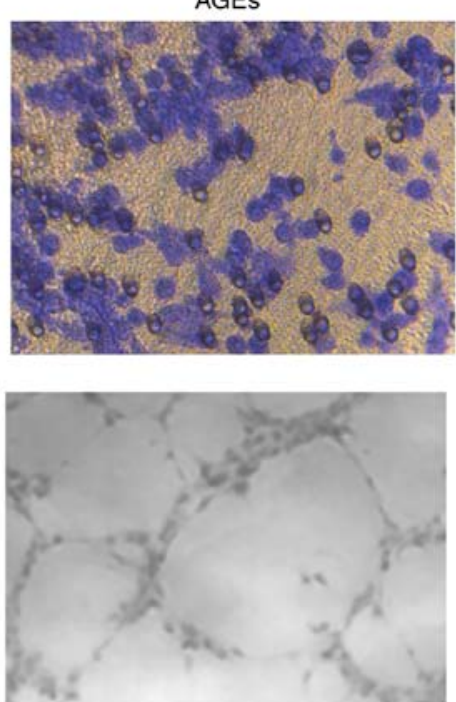

D

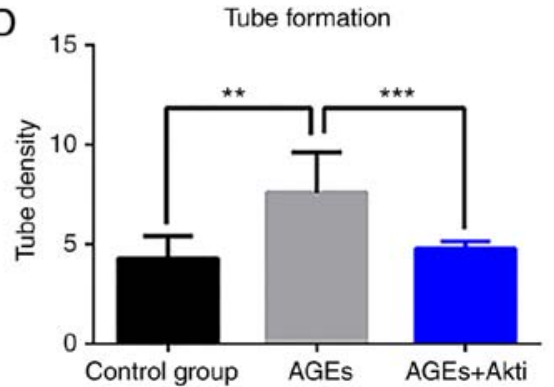

Figure 5. Invasive and tube forming ability of HRCECs. (A) Representative results of HRCEC invasion in control group following AGE and Akti treatment. AGE treatment significantly increased HRCECs invasion, and Akt inhibitor reduced this effect. (B) The tube formation ability of HRCECs was measured in control HRCECs, AGEs treated HRCECs, and Akt inhibitor-treated HRCECs. Magnification, x200. (C) Differences in invasive and (D) tube formation ability were statistically significant. ${ }^{* * *} \mathrm{P}<0.01,{ }^{* * *} \mathrm{P}<0.001,{ }^{* * * * *} \mathrm{P}<0.0001$ vs. the AGEs treated group. HRCECs, human retinal capillary endothelial cells; AGE, advanced glycation end products; PI3K, phosphoinositide 3-kinase; Akt, protein kinase B; i, inhibitor.

Müller cells are a type of retinal glial cell that have been demonstrated to be critical to retinal development, by serving as promoters of retinal growth and histogenesis (13). HRCECs are the major cell type that causes vascularization in DR (14). In the present study; the effects of AGEs on these two cell types were examined. Initially, the expression of RAGEs in Müller cells and HRCECs following AGE treatment was investigated. These two cell types expressed a basal level of RAGEs without any treatment. When treated with AGEs, they expressed a much higher level of RAGEs, compared with 
the control group. The effects of AGEs on Müller cells and HRCEC viability were subsequently examined. AGE treatment significantly enhanced HRCEC viability. The PI3K/Akt pathway has critical roles in regulating cell proliferation and death, and has been demonstrated to be involved in a number of AGE-associated biological processes, including autophagy and cell migration $(15,16)$. When cells were treated with PI3Ki, however, no obvious alterations in cell viability were observed in Müller cells or HRCECs. When cells were treated with Akti, the viability of HRCECs was dramatically suppressed, whereas Müller cell viability was not influenced. The following cell proliferation and apoptosis assays confirmed the findings of the cell viability assay, and suggested that AGE facilitated the survival of HRCECs in an Akt-dependent way. These initial results suggested that the AGEs primarily influenced HRCECs, rather than Müller cells.

Retinal vascularization is a coordinated collaboration involving several cell types, including endothelial cells, pericytes and astrocytes, and a dynamic balance of positive and negative regulatory factors (17-19). In angiogenesis-associated DR, this delicate balance is disturbed (10). Since AGEs regulate HRCEC proliferation via Akt, whether or not AGEs regulate the vascularization of the retina was investigated. By measuring the expression of genes associated with angiogenesis, it was demonstrated that the AGE treatment induced a pro-angiogenic gene expression, including VEGF, VEGFR, FGF, Ang1, Ang2 and PDGF. In addition, PEDF expression was decreased. Treatment with Akti inhibited these effects. Furthermore, the HRCEC invasion and tube formation assays also indicated the Akt-dependent pro-angiogenic effects of AGEs.

Studies of HRCECs have shed light on the earliest stages of DR and other diseases of the retinal microvasculature (20-22). However, whether or not the PI3K/Akt pathway influences AGE-mediated DR development remains unclear. The present study reported that AGEs induced HRCEC proliferation and angiogenesis via an Akt-dependent mechanism. Inhibiting the Akt pathway prevented the effects of AGEs on HRCEC proliferation and vascularization. Therefore, targeted therapies that suppress Akt function may be a promising treatment for retinal vascularization-associated diseases, including DR.

\section{Acknowledgements}

The authors acknowledge the support from the First People's Hospital of Yunnan Province during the present study.

\section{Funding}

The present study was supported by the Health Science Program of Yunnan Province (grant no. 2016NS238) and the Health Science Profession Training Program of Kunming, Yunnan Province (grant no. 2016-sw-66).

\section{Availability of data and materials}

The datasets used and/or analyzed during the present study are available from the corresponding author on reasonable request.

\section{Authors' contributions}

DT was responsible for study design, major experiments, data analysis and manuscript preparation. NN was responsible for experiments, data analysis and manuscript preparation. TZ conducted some experiments, and performed data analysis and manuscript preparation. CL was responsible for literature review, data analysis, and manuscript preparation and revision. QS was responsible for data interpretation, manuscript revision, and data collection. LW performed some experiments and was responsible for manuscript preparation and revision. YM was responsible for funding collection, study design and manuscript revision.

\section{Ethics approval and consent to participate}

Not applicable.

\section{Patient consent for publication}

Not applicable.

\section{Competing interests}

The authors declare that they have no competing interests.

\section{References}

1. Tripathi BK and Srivastava AK: Diabetes mellitus: Complications and therapeutics. Med Sci Monit 12: RA130-RA147, 2006.

2. Bos M and Agyemang C: Prevalence and complications of diabetes mellitus in Northern Africa, a systematic review. BMC Public Health 13: 387, 2013.

3. Vlassara $\mathrm{H}$ and Uribarri J: Advanced glycation end products (AGE) and diabetes: Cause, effect, or both? Curr Diab Rep 14: 453, 2014

4. Nowotny K, Jung T, Höhn A, Weber D and Grune T: Advanced glycation end products and oxidative stress in type 2 diabetes mellitus. Biomolecules 5: 194-222, 2015.

5. Xie J, Méndez JD, Méndez-Valenzuela V and AguilarHernández MM: Cellular signalling of the receptor for advanced glycation end products (RAGE). Cell Signal 25: 2185-2197, 2013.

6. Ott C, Jacobs K, Haucke E, Navarrete Santos A, Grune T and Simm A: Role of advanced glycation end products in cellular signaling. Redox Biol 2: 411-429, 2014.

7. Toker A and Marmiroli S: Signaling specificity in the Akt pathway in biology and disease. Adv Biol Regul 55: 28-38, 2014.

8. Jacot JL and Sherris D: Potential therapeutic roles for inhibition of the PI3K/Akt $/ \mathrm{mTOR}$ pathway in the pathophysiology of diabetic retinopathy. J Ophthalmol 2011: 589813, 2011.

9. Yau JW, Rogers SL, Kawasaki R, Lamoureux EL, Kowalski JW, Bek T, Chen SJ, Dekker JM, Fletcher A, Grauslund J, et al: Global prevalence and major risk factors of diabetic retinopathy. Diabetes Care 35: 556-564, 2012.

10. Tarr JM, Kaul K, Chopra M, Kohner EM and Chibber R: Pathophysiology of diabetic retinopathy. ISRN Ophthalmol 2013: 343560, 2013

11. Antonetti DA, Klein R and Gardner TW: Diabetic retinopathy. N Engl J Med 366: 1227-1239, 2012.

12. Zong H, Ward M and Stitt AW: AGEs, RAGE, and diabetic retinopathy. Curr Diab Rep 11: 244-252, 2011

13. Goldman D: Müller glial cell reprogramming and retina regeneration. Nat Rev Neurosci 15: 431-442, 2014.

14. Shin ES, Sorenson CM and Sheibani N: Diabetes and retinal vascular dysfunction. J Ophthalmic Vis Res 9: 362-373, 2014.

15. Qin Q, Niu J, Wang Z, Xu W, Qiao Z and Gu Y: Heparanase induced by advanced glycation end products (AGEs) promotes macrophage migration involving RAGE and PI3K/AKT pathway. Cardiovasc Diabetol 12: 37, 2013. 
16. Hou X, Hu Z, Xu H, Xu J, Zhang S, Zhong Y, He X and Wang N: Advanced glycation endproducts trigger autophagy in cadiomyocyte Via RAGE/PI3K/AKT/mTOR pathway. Cardiovasc Diabetol 13: 78, 2014.

17. Smith LE, Shen W, Perruzzi C, Soker S, Kinose F, Xu X, Robinson G, Driver S, Bischoff J, Zhang B, et al: Regulation of vascular endothelial growth factor-dependent retinal neovascularization by insulin-like growth factor-1 receptor. Nat Med 5: 1390-1395, 1999.

18. Majka S, McGuire PG and Das A: Regulation of matrix metalloproteinase expression by tumor necrosis factor in a murine model of retinal neovascularization. Invest Ophthalmol Vis Sci 43: 260-266, 2002.

19. Gupta N, Mansoor S, Sharma A, Sapkal A, Sheth J, Falatoonzadeh P, Kuppermann B and Kenney M: Diabetic retinopathy and VEGF. Open Ophthalmol J 7: 4-10, 2013.

20. Lois N, McCarter RV, O'Neill C, Medina RJ and Stitt AW: Endothelial progenitor cells in diabetic retinopathy. Front Endocrinol (Lausanne) 5: 44, 2014.
21. Joussen AM, Murata T, Tsujikawa A, Kirchhof B, Bursell SE and Adamis AP: Leukocyte-mediated endothelial cell injury and death in the diabetic retina. Am J Pathol 158: 147-152, 2001.

22. Kady N, Yan Y, Salazar T, Wang Q, Chakravarthy H, Huang C, Beli E, Navitskaya S, Grant M and Busik J: Increase in acid sphingomyelinase level in human retinal endothelial cells and CD34+ circulating angiogenic cells isolated from diabetic individuals is associated with dysfunctional retinal vasculature and vascular repair process in diabetes. J Clin Lipidol 11: 694-703, 2017.

This work is licensed under a Creative Common

Attribution-NonCommercial-NoDerivatives 4.0 International (CC BY-NC-ND 4.0) License. 